\title{
Lab Mice in the Field: Unorthodox Daily Activity and Effects of a Dysfunctional Circadian Clock Allele
}

\author{
Serge Daan, ${ }^{*, 1,2}$ Kamiel Spoelstra, ${ }^{*, 2,3}$ Urs Albrecht, ${ }^{\dagger}$ Isabelle Schmutz, ${ }^{\dagger}$ Moritz Daan, ${ }^{\ddagger}$ Berte Daan, ${ }^{\$}$ \\ Froukje Rienks, "Inga Poletaeva, " Giacomo Dell'Omo, ${ }^{\#, 4}$ Alexei Vyssotski, ${ }^{\#, 5}$ and Hans-Peter Lipp ${ }^{\#}$ \\ ${ }^{*}$ Centre for Life Sciences, University of Groningen, The Netherlands, ${ }^{+}$Department of Medicine, \\ University Fribourg, Switzerland, ${ }^{\ddagger}$ University of Leiden, The Netherlands, ${ }^{\circledR}$ ETH Zürich, Switzerland, \\ "Netherlands Institute of Ecology, Nieuwersluis, The Netherlands, "Laboratory of Physiology and \\ Genetics of Behavior, Moscow State University, Moscow, Russia, and "Division of Neuroanatomy \\ and Behavior, Institute of Anatomy, University of Zürich, Switzerland
}

\begin{abstract}
Daily patterns of animal behavior are potentially of vast functional importance. Fitness benefits have been identified in nature by the association between individual timing and survival or by the fate of individuals after experimental deletion of their circadian pacemaker. The recent advances in unraveling the molecular basis of circadian timing enable new approaches to natural selection on timing. The investigators report on the effect and fate of the mutant $\operatorname{Per} 2^{\text {Brdm } 1}$ allele in 4 replicate populations of house mice in a seminatural outside environment over 2 years. This allele is known to compromise circadian organization and entrainment and to cause multiple physiological disturbances. Mice $(N=250)$ bred from Per ${ }^{\text {Brdm } 1}$ heterozygotes were implanted subcutaneously with transponders and released in approximately Mendelian ratios in four $400 \mathrm{~m}^{2}$ pens. An electronic system stored the times of all visits to feeders of each individual. The study first demonstrates that mice are not explicitly nocturnal in this natural environment. Feeding activity was predominantly and sometimes exclusively diurnal and spread nearly equally over day and night under the protective snow cover in winter. The effect of Per2 ${ }^{\text {Brdm } 1}$ on activity timing is negligible compared to seasonal changes in all genotypes. Second, the Per2 ${ }^{B r d m 1}$ allele did not have persistent negative effects on fitness. In the first year, the allele gradually became less frequent by reducing survival. New cohorts captured had the same Per ${ }^{B r d m 1}$ frequency as the survivors from previous cohorts, consistent with an absence of an effect on reproduction. In the second year, the allele recovered to about its initial frequency (0.54). These changes in selective advantage were primarily due to female mice, as females lived longer and the sex ratio dropped to about $25 \%$ males in the population. While it is unknown which selective advantage led to the recovery, the results caution against inferences from laboratory experiments on fitness consequences in the natural environment. It also demonstrates that the activity of mice, while strictly nocturnal
\end{abstract}

\footnotetext{
1. To whom all correspondence should be addressed: Serge Daan, Centre for Life Sciences, University of Groningen, Hoofdweg 274, 9765 CN Paterswolde, The Netherlands; e-mail: s.daan@rug.nl.

2. These authors contributed equally to this work.

3. Present address: Netherlands Institute of Ecology, Heteren, The Netherlands.

4. Present address: Ornis Italica, I-00199 Rome, Italy.

5. Present address: Institute of Neuroinformatics, University of Zürich/ETH Zürich, Switzerland.
} 
in the laboratory, may be partially or completely diurnal in the field. The new method allows assessment of natural selection on specific alleles on a day-today basis.

Keywords mice, Per2, population, natural activity, survival, selection control

Life has evolved on a rotating planet. This has led to the ubiquitous capacity of animals to program their behavior in adaptation to the 24-h rhythm of the environment. They achieve this by endogenous circadian oscillators entrained by the 24-h light-dark cycle such that the behavioral rhythms they drive maintain a stable phase relationship with day and night. It is generally accepted that natural selection has tuned - and continues to tune- the underlying physiological mechanisms by favoring optimal daily timing (e.g., Pittendrigh, 1960). The search for these mechanisms is now a worldwide endeavor at all levels from molecular genetics to behavior and cognition.

In contrast, studies assessing the presumed selective premium on daily timing are remarkably few. Such studies have made use of spontaneous natural variation in behavioral timing in natural populations directly affecting individual mortality in birds (Daan and Tinbergen, 1980). There are also the heroic efforts of DeCoursey and colleagues (2000), who studied the consequences for survival in the wild of ablations of the circadian pacemaker in the suprachiasmatic nuclei of chipmunks. These studies indicate major negative effects of deviating phase or compromised circadian organization on fitness components. One might thus expect a reduced rate of propagation of alleles impairing circadian organization. The recent surge of circadian molecular genetics opens up a perspective for more direct approaches to natural selection. One important study has demonstrated that the match between natural endogenous period of mutants and that of the entraining light-dark cycle is crucial for a sharp selective advantage in strains of Cyanobacteria (Ouyang et al., 1998). The availability of mutant mouse strains with targeted functional deletions of genes involved in circadian organization allows us to take a similar approach in higher vertebrates.

We have taken up this challenge in a study in a seminatural setting outdoors. Our purpose is 2-fold. First, we aim to obtain circadian behavior patterns in standard laboratory mouse (mixed background: C57BL/6-129 SvEvBrd) in this seminatural environment. Knowledge of the behavior patterns of mice in the field is limited. We were interested to see how these compare with the wheel running activity pattern that is used so widely in the laboratory as an expression of the circadian clock system. We included mutant mice with a functional deletion of the $m P e r 2$ gene known to modify circadian behavior in the laboratory (Albrecht et al., 2007; Zheng et al., 1999). Per $2^{\text {Brdm1 }}$ mice show a shortened circadian period in DD and eventually become arrhythmic (Zheng et al., 1999). In LL they display a shortening instead of lengthening of period (Spoelstra and Daan, 2008; Steinlechner et al., 2002). Resetting behavior is impaired in these animals (Albrecht et al., 2001; Spoelstra et al., 2004). They entrain to LD 12:12, but long photoperiods fail to compress activity into the short night (Comas, 2009). There is no anticipatory activity preceding feeding time in Per2 ${ }^{\text {Brdm } 1}$ mice (Feillet et al., 2006; Mendoza et al., 2010). Homozygous Per ${ }^{\text {Brdm } 1}$ mutants are also characterized by a series of health-related deficits (see Albrecht et al., 2007 for review). The second aim is to assess the fate of the allele in isolated mice populations exposed to this environment through the natural seasons. We asked whether the mutation causing disturbances in the circadian system and physiology would indeed entail negative selection in a seminatural setting.

The study employed 4 large outdoor pens in a remote woodland area in Western Russia. This facility was previously built and used by one of us (H-P.L.) to study the consequences of genetic cognitive deficits for behavior in mice (Abbott, 2007; Vyssotski et al., 2002). Mice $(N=250)$ bred from heterozygote pairs were released after being equipped with subcutaneous transponders. The transponders with individual recognition allowed us to continuously record visits to feeders surrounded by a circular antenna hooked up to a central recording system. The records allow the reconstruction of daily feeding activity patterns both individually and for the populations. They also enable us to assess the date of the final record for each individual and by inference of the differential rates of mortality of different genotypes. After the initial release, all mice were live-trapped on 4 subsequent occasions through the 2 years of the study, and all new mice were provided with transponders. All mice were genotyped as wild-type, heterozygous, or homozygous for the mutant Per $2^{\text {Brdm1 }}$ allele. Their relative proportions in the 4 populations make it possible to reconstruct the rates of mortality and new recruitments to the population. The data thus give us at least partial answers to 
the 2 questions raised on activity patterns and on potential fitness consequences of a circadian clock allele.

\section{METHODS}

Two hundred and fifty mice were bred from pairs heterozygous for Per $2^{\text {Brdm1 }}$ in mixed background of C57BL/6, 129SvEvBrd (see Albrecht et al., 2001) at the Centre for Life Sciences in Haren, The Netherlands. The offspring were either wild-type, heterozygous, or homozygous for the mutation, henceforth designated as Per $2^{++}$, Per $2^{+\mathrm{m}}$ and Per $2^{\mathrm{mm}}$, respectively. At the average age of 63 days (SD, 5.4) they were lightly anesthetized with methoxyflurane and subcutaneously injected under the skin of the back with glass-covered microtransponders (11.5 x $2.2 \mathrm{~mm}$; Trovan ID100) by means of a sterile hypodermic needle. Genotypes were assessed in the laboratory of U.A. in Fribourg from 3-mm tail tips (see supplementary online materials [SOM]-1). The mice were then transferred to Russia. Upon arrival at the field station Chisti Lec (Clear Forest), Bubonizi (Pozhnia, Tver Region, Western Russia, 5644'7.99'”N; $\left.31^{\circ} 31^{\prime} 34.44^{\prime \prime} \mathrm{E}\right)$ they were released into 4 large outside enclosures on May 21,2005, at the average age of 76 days $(\mathrm{SD}, 5.4)$. Each of these pens received 62 or 63 mice to a total of 250. The distribution of sexes and genotypes at release and during 4 later retrappings is shown in Table 1. There was a slight initial excess of Per $2^{\text {Brdm1 }}$ alleles in the original population, but the distribution (54 wildtype, 120 heterozygote, 76 homozygote mutants) did not deviate significantly from the expected Mendelian $1: 2: 1$ proportion $\left(\chi^{2}=4.272 ; \mathrm{df}=2\right)$. Males $(n=129)$ slightly outnumbered females $(n=121)$.

Each experimental pen measured $20 \times 20 \mathrm{~m}$ and was surrounded by a slate wall, $1 \mathrm{~m}$ high and sunk $50 \mathrm{~cm}$ into the soil. The top of the wall was covered by smooth zinced iron. The pens were organized to allow for interindividual competition for food, water, shelter, and mating as well as for exposure to natural weather conditions and aerial predation. Terrestrial predators were kept away by means of an electrically charged wire on top of the walls, as used for cattle fences (but see Results). The pens were not covered, and aerial predators had free access. In each pen, 2 closed shelters of $2 \times 4 \mathrm{~m}$ and 70 $\mathrm{cm}$ depth filled with hay, straw, and branches provided opportunities to escape aggressive encounters. The initial release of the mice was in these shelters. Wood covered runways from the shelters led to 2 feeders in each pen, consisting of a tray filled ad libitum with standard lab chow under a wooden roof. The food and two 1-L water bottles near the feeders were checked and replenished at irregular times once per 2 to 3 days. A circular antenna loop mounted watertight in a PVC pipe surrounded the food tray. The 8 antennas from all pens were connected to 1 data storage device (UKID System, Collinson \& Co., Caterall, Preston, UK) in a separate central shed. This scanned the antennas continuously for 2 years with 3 interruptions, 1 for a week in early June 2005 due to a lightning storm, 1 in August 2006, and 1 in January 2007 in extremely cold weather (see Figure 1).

We trapped all mice in each pen on 4 occasions: September 14-22, 2005, May 8-13, 2006, August 2-20, 2006, and May 11-16, 2007. Each time, transponders were read externally from those mice carrying one, and all new mice born in the preceding interval received a transponder except at the final capture. Tail tips were collected as before and shipped to Fribourg for genotyping. All mice captured were maintained in standard cages, either separately (males) or group housed (females). They were released again in the same pen after all mice known to be alive from the activity records had been trapped and traps remained empty for at least 1 day. After the final trapping episode in May 2007, all mice were sacrificed by cervical dislocation under methoxyflurane anesthesia. Genotyping for Per $2^{\text {Brdm1 }}$ was done in all mice released and trapped. All experiments were carried out in accordance with national regulations (Rules of Ministry of Health Russia, 1977; EU directive 86; permit to H-PL from the Swiss National Science Foundation).

\section{RESULTS}

The mouse populations in the pens survived over the 2 years, except in pen 2. In this pen, the whole population was wiped out in February 2006 when accumulated snow drift in one corner allowed a European mink (Mustela lutreola) to enter the pen over the fence. The predator was sighted and trapped in pen 2 after the last of the mice had been killed here. Thus, the remaining 15 months of the study were restricted to pens 1,3 , and 4 . The average number of mice per pen was 62.5 at release and 56.7, 52.0, 57.3, and 66.7 at the 4 consecutive retrappings (see Table 1 ). Thus, the populations retained stable size in spite of the restricted shelter space and feeding sites.

\section{Feeding Activity}

Upon release in the enclosures, mice rapidly found the feeders and left records of their visits in the recording system. Of 250 mice released initially (cohort 1), 4 never left a record. For the other 246 mice, the number 
Table 1. Genotype and sex distribution of mice at release and in 4 consecutive complete recaptures.

\begin{tabular}{|c|c|c|c|c|c|c|c|c|c|}
\hline \multirow{2}{*}{$\begin{array}{l}\text { Date } \\
\text { Cohort }\end{array}$} & \multirow{2}{*}{$\begin{array}{c}22 \\
\text { May } \\
2005 \\
1 \\
\end{array}$} & \multicolumn{2}{|c|}{$\begin{array}{c}14-22 \\
\text { September } \\
2005\end{array}$} & \multicolumn{2}{|c|}{$\begin{array}{l}8-13 \\
\text { May } \\
2006\end{array}$} & \multicolumn{2}{|c|}{$\begin{array}{c}2-20 \\
\text { August } \\
2006\end{array}$} & \multicolumn{2}{|c|}{$\begin{array}{l}\text { 11-16 } \\
\text { May } \\
2007\end{array}$} \\
\hline & & 1 & 2 & $1-2$ & 3 & $1-3$ & 4 & $1-4$ & 5 \\
\hline F Per2 ${ }^{++}$ & 28 & 11 & 19 & 12 & 20 & 8 & 21 & 5 & 24 \\
\hline M Per2 $^{++}$ & 26 & 3 & 12 & 4 & 14 & 3 & 23 & 1 & 22 \\
\hline F Per2 ${ }^{+m}$ & 53 & 25 & 45 & 19 & 38 & 24 & 34 & 15 & 55 \\
\hline M Per2 $^{+m}$ & 67 & 7 & 38 & 8 & 20 & 2 & 27 & 3 & 31 \\
\hline F Per2 ${ }^{\mathrm{mm}}$ & 40 & 13 & 35 & 5 & 9 & 8 & 12 & 6 & 22 \\
\hline M Per2 $2^{\mathrm{mm}}$ & 36 & 6 & 13 & 1 & 6 & 2 & 8 & 1 & 15 \\
\hline Total & 250 & 65 & 162 & 49 & 107 & 47 & 125 & 31 & 169 \\
\hline$\%$ Per2 $^{\text {Brdm1 }}$ & 54.4 & 53.8 & 55.2 & 39.8 & 41.1 & 48.9 & 40.4 & 51.6 & 47.3 \\
\hline$\chi^{2}(d f=2)$ & & \multicolumn{2}{|c|}{0.17} & \multicolumn{2}{|c|}{0.09} & \multicolumn{2}{|c|}{2.32} & \multicolumn{2}{|c|}{0.89} \\
\hline
\end{tabular}

New individuals are indicated in bold type by cohorts $1-5$; previously marked individuals by cohort groups $(1,1-2,1-3,1-4)$. Sexes are indicated by $M$ and $F$. The bottom rows show the frequency of Per $2^{\text {Brdm1 }}$ in old and new cohorts and the (all nonsignificant) $\chi^{2}$ values for the differences between genotype distribution of old cohort survivors against new cohorts caught in the same trapping session.

Table 2. Mean recording time (days from release till last record), and activity counts per day of the 3 genotypes in cohort 1, released 20 May 2005.

\begin{tabular}{|l|c|c|c|c|c|}
\hline \multirow{2}{*}{} & \multicolumn{2}{|c|}{ Recording Time (Days) } & \multicolumn{2}{c|}{ Activity/Day } & \multirow{2}{*}{$n$} \\
\cline { 2 - 5 } & Mean (SEM) & Range & Mean (SEM) & Range & $n$ \\
\hline F Per2 $^{++}$ & $115(26)$ & $6-410$ & $80(16)$ & $6-319$ & 28 \\
\hline M Per2 $^{++}$ & $42(11)$ & $4-202$ & $45(21)$ & $1-536$ & 26 \\
\hline F Per2 $^{+m}$ & $150(23)$ & $4-584$ & $46(6)$ & $2-177$ & 53 \\
\hline M Per2 $^{+m}$ & $30(8)$ & $2-355$ & $28(5)$ & $1-178$ & 65 \\
\hline F Per2 $^{\text {mm }}$ & $95(17)$ & $4-479$ & $56(8)$ & $2-181$ & 40 \\
\hline M Per2 $^{\text {mm }}$ & $46(9)$ & $3-151$ & $13(4)$ & $0-111$ & 34 \\
\hline F all & $124(13)$ & $4-584$ & $57(6)$ & $2-319$ & 121 \\
\hline M all & $37(5)$ & $2-355$ & $28(5)$ & $0-536$ & 125 \\
\hline
\end{tabular}

Figures in parentheses are standard errors of the mean of the individual values. $\mathrm{F}$ and $\mathrm{M}$ indicate females and males. ANOVA: Recording time: effect of sex, $F=30.2(p<0.001)$; effect of genotype, $F=0.77$ (NS). Activity: effect of sex, $F=15.7(p<0.001)$; effect of genotype, $F=4.20(p<0.02)$

of individual records varied from 1 to 23,393 (mean 2600). The last record was on average 80 days after release (range, 2-584 days), corresponding with an average age of 156 days. Females lived on average about 3 times as long as males, while genotype did not contribute to the explained variance in recording time or life span (Table 2). Mice were recorded at the feeder on average 42 times per $24 \mathrm{~h}$ (range, 0.3-536). Females showed on average twice as many activity records per day as males. Per 2 mutant alleles were associated with less activity per day (Table 2).

The overall daily distribution of activity of all mice in the study is summarized in 3 composite doubleplotted actograms in Figure 1, 1 for each genotype. We see that there is no restriction of activity to the night. Instead, there are pronounced variations in the course of the year: episodes with mainly diurnal activity, times with mainly nocturnal activity, and times with activity throughout day and night. These changes are not due to new cohorts replacing the old ones but are seen likewise in records of individual animals. Figure 2 shows records for 2 individual mice, 1 wild-type and 1 homozygote Per 2 mutant. These were selected because of their exceptionally long activity records: Mouse no. 129 (female Per $2^{++}$) stayed alive in the enclosure for 387 days, mouse no. 25 (female Per ${ }^{\mathrm{mm}}$ ) for 261 days. Neither of these mice shows pronounced nocturnal concentration of activity. Both exhibited as much activity during daylight as during the night, except during a few hours after dawn in the wild-type mouse: this brief rest episode was consistently present throughout the summer. Both made a conspicuous seasonal switch toward nearly exclusively diurnal activity in the autumn of 2005, similar to the switch seen in the whole population (Figure 1). The diurnal activity disappeared again to make place for nearly arrhythmic patterns in both mice during the winter months, which represents activity under the snow. The difference between the patterns in the summer of 2005 of the 2 genotypes suggests that the homozygous Per2 ${ }^{\mathrm{mm}}$ mutation rendered mouse no. 25 arrhythmic even under seminatural conditions. Yet in autumn, we observed no difference in the patterns: both rhythms appear to be excellently entrained with activity only during the day.

Daily activity profiles (in records per individual mouse present per hour) are provided per genotype for the months January, April, July, and October in each of the 2 years in SOM-2. The extent of concentration of activity in daytime can be expressed in the Diurnality index $D$, originally introduced for voletrapping records (Hoogenboom et al., 1984): $D=\left(C_{d}\right.$ $\left.-C_{n}\right) /\left(C_{d}+C_{n}\right)$, where $C_{d}$ and $C_{n}$ are the activity counts per hour in daytime and at night (defined by civil twilights at dawn and dusk), respectively. Note that $D$ is a population as well as an individual measure: when population activity counts are expressed per 
all per2 ${ }^{++}$mice $(n=163)$

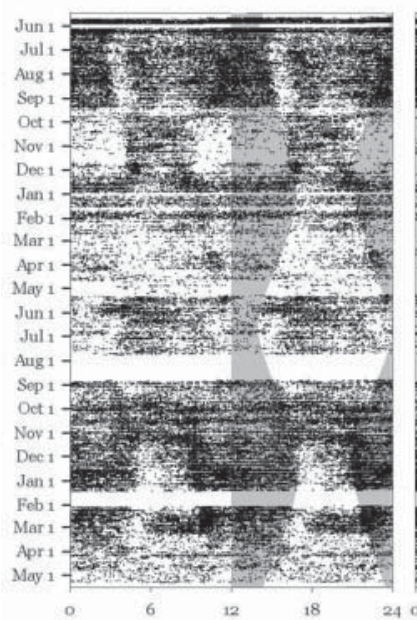

all per $^{+m}$ mice $(n=318)$

all per2 ${ }^{\mathrm{mm}}$ mice $(\mathrm{n}=158)$

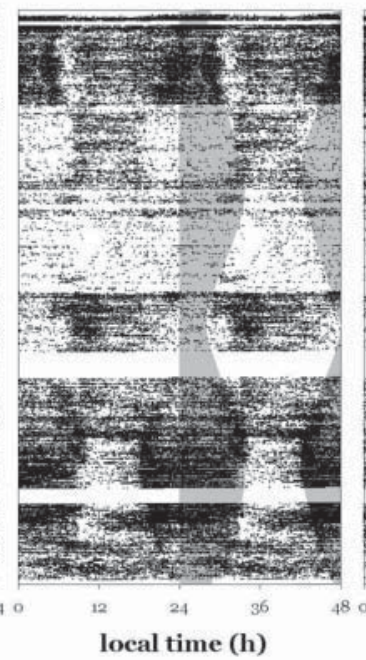

Figure 1. Composite activity records of all mice belonging to 3 genotypes. (A) Per2 ${ }^{++}$. (B) Per2 ${ }^{+m}$. (C) Per2 ${ }^{\mathrm{mm}}$. Gray area in right half of each double plot indicates night as defined by civil twilight (sun more than $6^{\circ}$ below horizon).

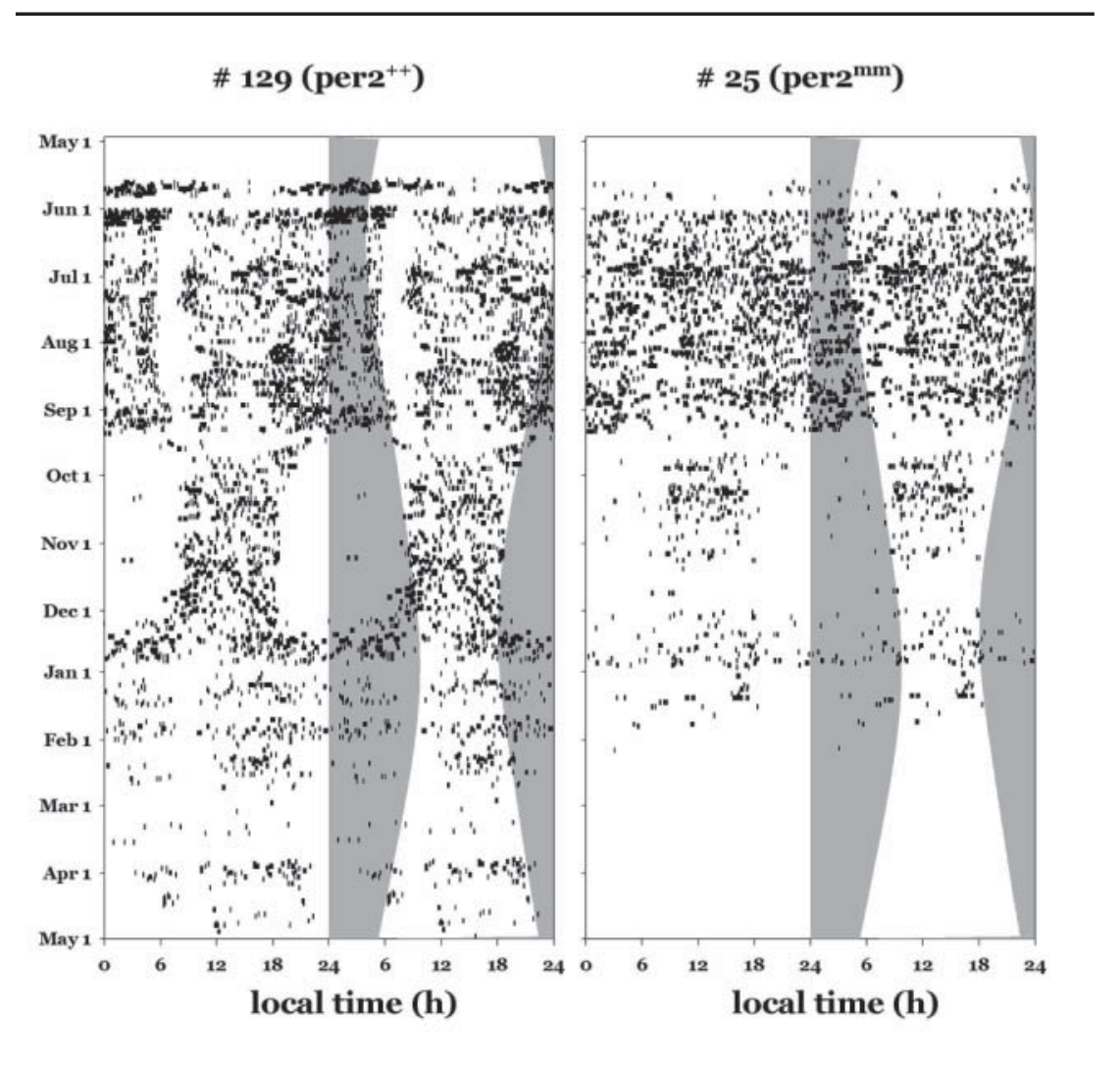

Figure 2. Individual activity records of 2 representative examples of a female wild-type (left) and a female homozygous Per2 mutant (right) mouse in seminatural conditions outdoors. Time span: May 1, 2005 to May 1, 2006. Gray area in right half of each double plot indicates night as defined by civil twilight (sun more than $6^{\circ}$ below horizon).

individual present $(n)$, both $C_{d}$ and $C_{n}$ are divided by $n$, and $D$ remains the same. Interindividual variance is not used for statistical testing. Figure 3 shows the seasonal changes in $D$ for May 2005 till February 2007 for the 3 genotypes separately. The expectation on the basis of the predominant nocturnality of mice in the laboratory would be that the index $D$ is close to -1 most of the time. In contrast, $D$ oscillated seasonally, with high levels around +0.7 in the autumn of 2005 and summer of 2006 and climbing again in the spring of 2007. Lower levels, with a slight dominance of nocturnal activity, around -0.3 were observed in the first summer and in the winters of 2006 and 2007. The drop in $D$ below zero (equal distribution over night and day) occurred in both winters close to the onset of snow cover and mean daily temperatures below zero (Figure 3B). In spring, positive $D$ values coincided more or less with the disappearance of snow cover. The patterns were very similar for the 3 genotypes, and for males and females separately (SOM$3)$. We tested monthly $D$ values per sex and genotype for effects of these factors as well as of mean air temperature $\left({ }^{\circ} \mathrm{C}\right.$ ) and snow cover (fraction of month with snow). The latter 2 were derived from the nearby meteorological station at Velikie Luki $\left(56^{\circ} 35^{\prime} \mathrm{N} ; 30^{\circ} 6^{\prime} \mathrm{E}\right)$. In the GLM model (SPSS) snow cover turned out to have an overriding significant effect (Wald $\chi^{2}=11.15 ; p=0.001$ ), without significant additional effects of sex, genotype (presence or absence of Per2 $^{+}$), temperature, or their interactions. In an ANOVA using month as a random factor, both sex and genotype (presence or absence of Per2 ${ }^{+}$) proved to contribute significantly to the explained variance in D. Males were slightly more diurnal than females and homozygous mutants 

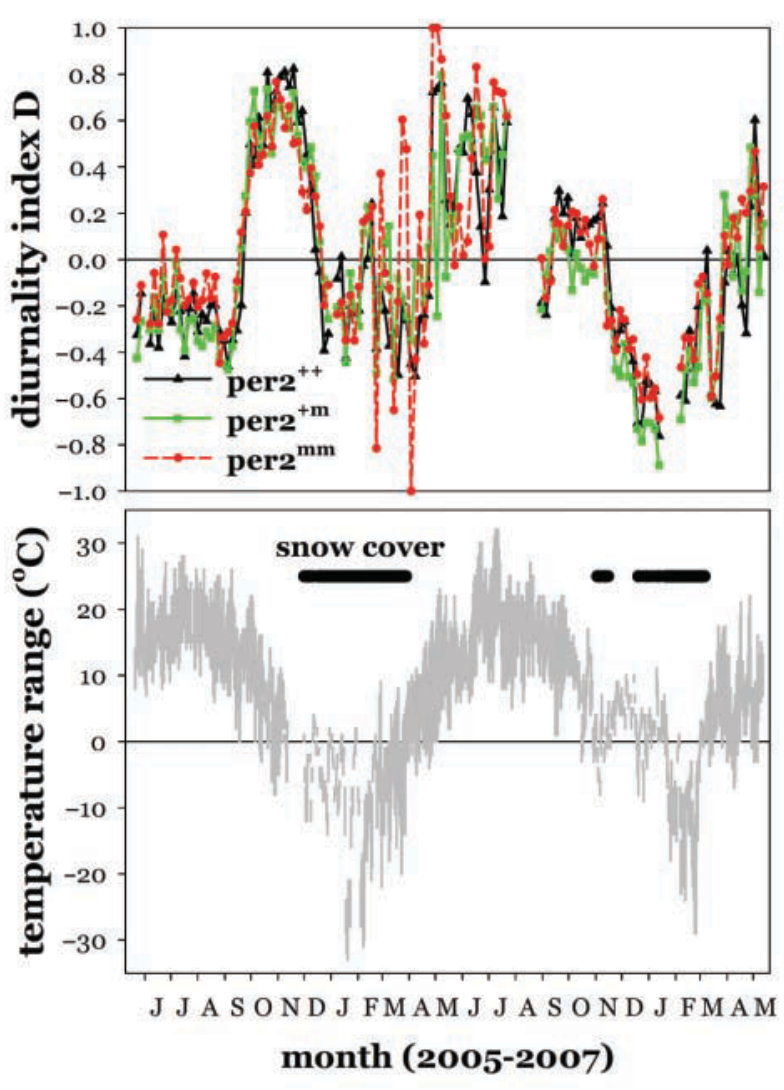

Figure 3. Upper panel: Diurnality index $D$ in each of the 3 genotypes during the study. Lower panel: Air temperature records from the nearby weather station Velikie Luki $\left(56^{\circ} 21^{\prime} \mathrm{N} ; 30^{\circ} 43^{\prime} \mathrm{E}\right)$.

slightly more diurnal than heterozygotes and wildtypes together. These differences were quantitatively negligible compared to the steep seasonal changes (SOM-3). The overriding seasonal variation of positive $D$ in the summer and negative $D$ in winter under the snow is only broken by the initial negative values observed in the summer of 2005. This is possibly related to the low initial density of mice.

\section{Differential Survival}

We assume that the final recording of each mouse represented the last day alive for this individual, with a margin of 1 week. This assumption is supported by the fact that all recaptured individuals had been recorded at the feeders during the last week before recapture. It allows us to establish survival curves for the 3 genotypes following release in the enclosures. In Figure 4, survival is plotted for 2 classes (the years 2005 and 2006) separately. These data include all individuals for which the last record was obtained at least 10 days after release, and thus excludes those that did not become established in the population. This is especially relevant for the first cohort, born in the laboratory, where 98 mice (39\% of the 250 released) disappeared in the first 10 days, after which the population remained relatively stable. The cohorts were pooled per year to obtain sufficient data sets. Figure 4 shows that homozygous Per $2^{\mathrm{mm}}$ disappeared faster from the population of 2005 than heterozygous and wild-type mice. The average mortality rates (in \% / day) are estimated from the regression slopes in Figure 4 at 0.449 (Per2 $\left.{ }^{\mathrm{mm}}\right), 0.278$ $\left(\right.$ Per2 $\left.{ }^{+m}\right)$, and $0.288\left(\right.$ Per2 $\left.^{++}\right)$, respectively. In 2006 we observe the opposite trend. In this year the wild-type (mortality $0.272 \%$ / day) and heterozygote mice $(0.260$ $\%$ /day) disappeared at a similar rate as in 2005, while homozygous Per $2^{\mathrm{mm}}$ mutants survived best (mortality $0.174 \%$ / day). The distributions for the 2006 year class are truncated by the termination of the study in spring 2007 , but this does affect the general conclusion. Indeed, median life spans were significantly dependent on genotype, albeit in opposite direction in the 2005 and 2006 year classes (SOM-4).

\section{Per ${ }^{\text {Brdm }}$ Allele Frequency}

The data provide a day-by-day assessment of the differential survival of the 3 genotypes in each of the 4 enclosures. The result is a change in the frequency of the Per ${ }^{\text {Brdm }}$ allele in each population. This analysis (Figure 5A) shows slight differences between the 4 pens. In pen 1 and 2, Per ${ }^{\text {Brdm }}$ frequency dropped from the starting frequency of 0.54 to about 0.25 over the first winter. The population in pen 2 was then extinguished by a European mink entering the pen in February 2006. In pen 1, the Per $2^{B r d m}$ frequency rose again over the second summer and winter until it had reached its original value again by the end of the study in spring 2007. The populations in pen 3 and pen 4 showed smaller changes, with a steady decrease from 0.54 to 0.35 in 2 years in pen 3 and a return after a first year drop from 0.54 to 0.45 back up to 0.55 in pen 4 . The $\operatorname{Per}^{\text {Brdm }}$ frequency in all pens together thus remained rather stable, with a drop of $14 \%$ during the first year compensated by an increase of $12 \%$ in $P e r 2^{\text {Brdm }}$ frequency over the second year.

Figure 5B provides the Per $2^{\text {Brdm }}$ frequency in the total population of the 4 pens and in each of the 2 sexes separately. The overall frequency now turns out to be composed of different patterns in the 2 sexes. The Per $2^{\text {Brdm }}$ frequency of males dropped sharply during the autumn of 2005 and remained stable around 0.35 from then onward till the end of the study. The frequency of the 


\section{year class 2005}

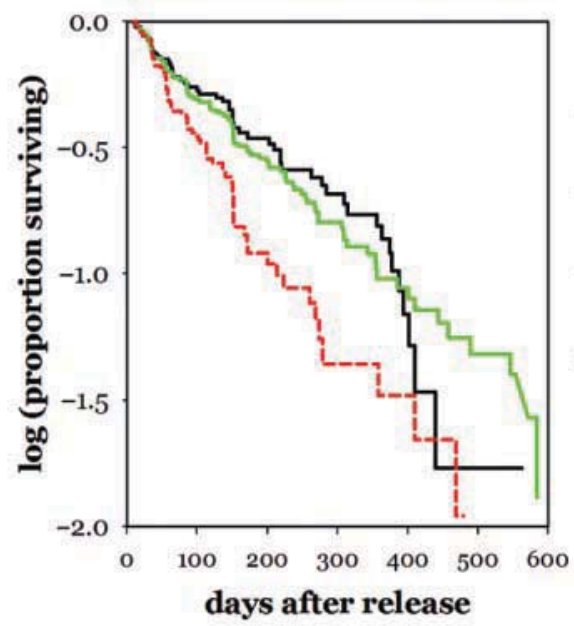

year class 2006

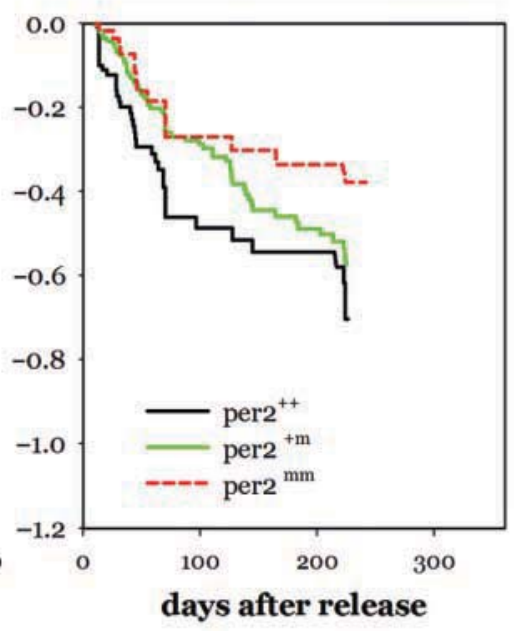

Figure 4. Survival curves for all mice of the 3 genotypes since the time of their release. (A) Mice born and released in 2005 (cohorts 1 and 2). The curves start at 10 days after release to exclude the individuals that did not become established in the field. (B) Mice captured and released in 2006 (cohorts 3 and 4). Note the difference in time scales on the abscissa: the class year of 2005 had 2 years to be recorded, the class year of 2006 only 1 year. The ordinate is scaled accordingly.

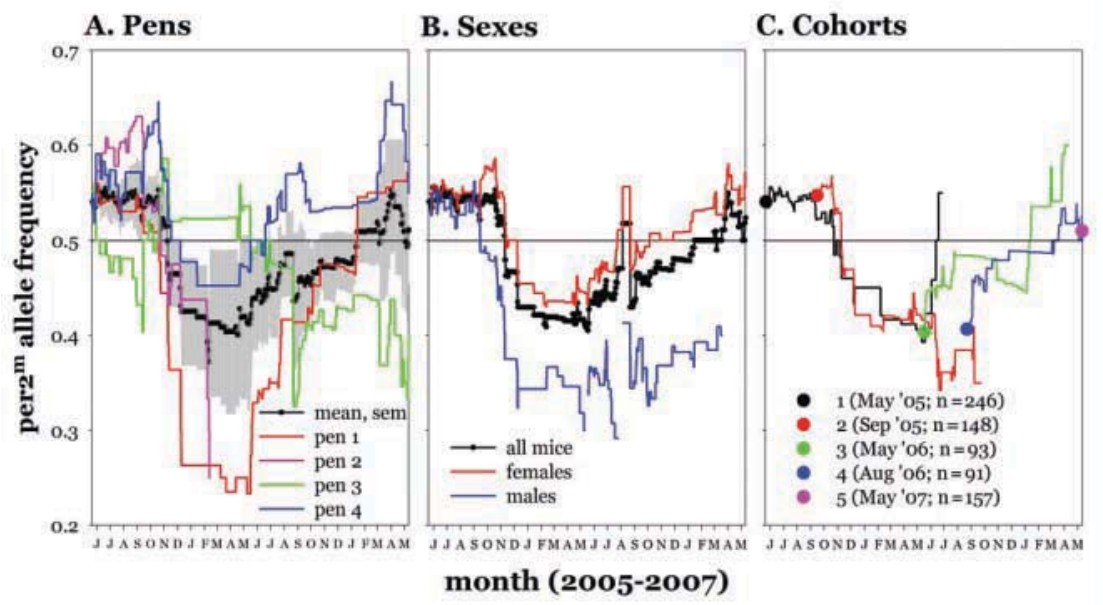

Figure 5. Day-to-day changes in the frequency of the Per2 ${ }^{\mathrm{Brdm} 1}$ allele in the populations over the 2 years of study. Gene frequencies were only calculated when 10 or more individuals were present. (A) The 4 pens separately (colors) and the mean of the 4 pens (black dots) \pm SEM (gray area). (B) The 2 sexes separately (colors) and combined (black dots). (C) The 5 cohorts separately (colors). Large symbols indicate the initial frequency in each cohort.

mutant Per2 gene in females dropped merely to 0.43 in the spring of 2006 and then increased steadily until the original level around 0.56 in the spring of 2007. By the highly skewed sex ratio in the population, the overall Per $2^{\text {Brdm }}$ frequency closely follows that in the females albeit at a level circa 2\% lower. Apparently, the initial deficit in fitness attributable to the Per $2^{\text {Brdm }}$ gene was primarily due to an effect in males. The sex ratio (percentage males) went sharply down in the very beginning of the study, probably due to intense competition between males, an effect observed in earlier outdoor studies (Van Zegeren, 1980). This change was particularly marked in mice homozygous for Per2 ${ }^{\text {Brdm }}$ (Figure 6).

Figure 5C presents the same data analyzed per cohort. In the first 2 cohorts, born in 2005 in the lab and field, respectively, Per ${ }^{\text {Brdm }}$ frequency declined in parallel over the first winter and summer. Cohorts 3 and 4 , both born in 2006, each showed a steady increase in Per $2^{\mathrm{Brdm}}$ frequency over the second year. In the end the initial selection against $\mathrm{Per} 2^{\mathrm{Brdm}}$ was nearly fully made up by its positive selection in the next generation. It is of interest that the initial Per $2^{\text {Brdm }}$ frequencies in each of the cohorts 2,3,4, and 5 were nearly equal to those in the surviving members of previous cohorts (see Figure 5C and Table 1). This suggests that changes in gene frequencies were not caused by differential reproduction but solely by differential survival.

\section{DISCUSSION}

The use of mice backcrossed from 2 inbred lab strains (C57Bl and 129) of course constrains the extrapolation to natural mouse populations. Also, for obvious reasons, the conditions under which our observations were made were neither as fully controlled as in the laboratory nor as fully natural as in the wild. Differences included exposure to predation, infectious diseases, and natural variations in temperature, humidity, and precipitation; competition for access to food, shelter, and mating; and interindividual aggression. Some of these conditions may be relevant to the differences between our results and those that might have been expected. Which conditions are most important, and just how they might cause differences between actual and expected results, is not obvious. 


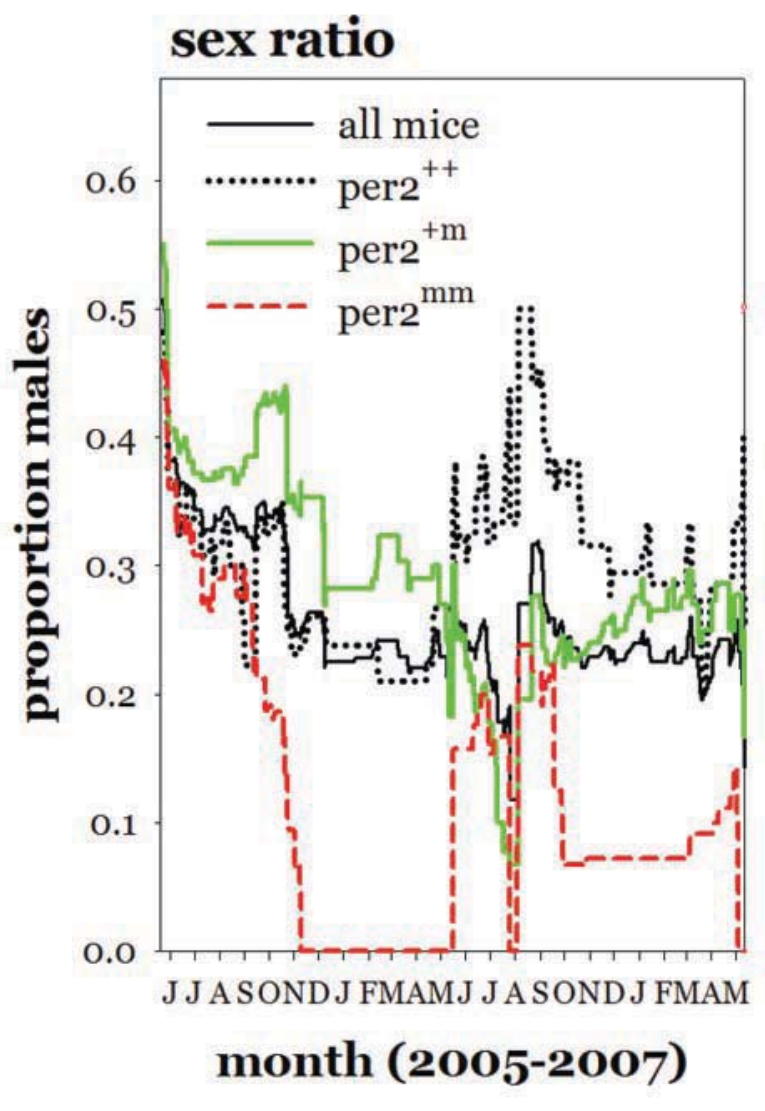

Figure 6. Changes in sex ratio in the 3 genotypes over the 2 years of the study.

This attests to the difficulty of extrapolating from the lab to the field.

\section{Activity Pattern of Mice under Natural Conditions}

The feeding activity of mice in 4 populations maintained in the outdoors enclosures was surprisingly prominent in daytime. In the laboratory, the same mouse strain is pronouncedly nocturnal. Nocturnal wheel running is used as the common readout of the circadian system in chronobiological studies worldwide. The main theory of entrainment by light is based on phase responses to brief exposures to light early and late in the day as are generally believed to represent natural exposure patterns (Pittendrigh and Daan, 1976). In the seminatural situation, feeding activity turns out to be much less restricted to the night. Throughout the summer we observed activity all through the day except in a window of a few hours following sunrise (Figures 2 and SOM-2). In the autumn of 2005 we observed nearly exclusively diurnal activity in the whole population. This led to a Diurnality index $D$-a parameter between -1 (exclusively nocturnal activity) and +1 (exclusive diurnality) - that approached +1 (Figure 3) At other times, under midwinter snow cover in both winters, activity was nearly randomly distributed over night and day ( $D$ values just below zero).

A more systematic seasonal change from nocturnality in summer to diurnality in winter is known from several vole (Microtus) species (Hoogenboom et al., 1984). In the montane vole, this is even observed in wheel running activity in the lab (Rowsemitt, 1991). The $D$ index increases in winter and reaches high values especially at high population density (Hoogenboom et al., 1984). The picture in mice is less systematic. In 2006, high $D$ values were observed during the summer, much earlier than in 2005. Hence, there is no simple relationship with ambient temperature (Figure 3). Possibly population density, which was initially very low in the summer of 2005 and higher in 2006 and 2007, has played a role. Be this as it may, the overall picture is that feeding often occurred more frequently in daytime than at night. Clearly the laboratory situation is not representative for the behavior of lab mice outside. A similar conclusion was recently drawn for Syrian hamsters, which are active outside their burrows in the wild in 2 episodes before dusk and after dawn and not at night (Gattermann et al., 2008). This is in sharp contrast with the pronounced nocturnal behavior of hamsters in the lab, even in feeding activity outside simulated burrow systems (Pratt and Goldman, 1986). The behavior of mammals differs between the lab and the field in many other respects (Calisi and Bentley, 2009).

One difference is that we recorded feeding activity, not wheel running. In the lab, feeding is less restricted to the night than running but still primarily nocturnal. The second difference is that mice in the lab are maintained on a continuous supply of food, for which they need not compete or even search, and they are thermally protected by maintenance in a stable ambient temperature above $20^{\circ} \mathrm{C}$. In our seminatural setting, food was also permanently available, but mice had to compete for access, with 2 feeders for about 60 mice per pen. Recent studies have shown that mice in the lab that have to work for their food show a gradual shift from nocturnal to diurnal activity (Hut et al., 2011). A similar shift was reported earlier specifically for the lactation period in reproducing females working for their food (Perrigo, 1987). The shift to diurnal activity is accompanied by nocturnal hypothermia (Hut et al., 2011; Schubert et al., 2010) and is most likely part of an energy savings strategy under metabolically challenging conditions. 
There is good evidence that these changes in activity phase do not involve a phase shift of the circadian pacemaker (Hut et al., 2011). It may well be that the use of the circadian activity rhythm as a readout of the pacemaker is valid only under ad libitum feeding conditions in the laboratory but not readily transferrable to the field. The reduced diurnality under winter snow cover may well reflect the reduced metabolic needs of the mice under protection and insulation by the snow. $D$ was much higher in the summer months, except during the first summer, when densities were initially low and may likewise have allowed reduced diurnality.

A third difference is that in the lab, males are the standard subjects in chronobiology. Our data refer to both sexes, but females dominated. House mouse demes generally consist of 1 male and several females, and males disappear sooner by interindividual aggressive interaction (Van Zegeren, 1980). This led to longer life spans in females than males (Table 2 and SOM-4) and low sex ratios in the population (Figure 6), in particular in the survivors from older cohorts (Table 1). Females had on average higher feeding activity (Table 2), which may be related to the increased energy needed during pregnancy and lactation (Schubert et al., 2009). The index of diurnality was significantly lower in females but only marginally so compared to the seasonal changes (SOM-3).

The Per $2^{\text {Brdm } 1}$ mutation had remarkably little impact on the activity rhythms observed in outdoor enclosures. In laboratory studies, differences in activity rhythms have been recorded primarily in constant conditions, where homozygous mutants have a much shorter free-running circadian period or show arrhythmia, and heterozygous mutants are similar to wild types with incidental bouts of arrhythmia (Zheng et al., 2001; Zheng et al., 1999). In a light-dark cycle they show reduced delay responses to evening light (Albrecht et al., 2001; Spoelstra et al., 2004) and, correspondingly, an advanced phase at least under long (summer) days (Comas, 2009). In the enclosures, the patterns of activity were not impressively different between the 3 genotypes. The low density of records of individual visits to the feeder (on average 42 per mouse per day) did not allow a precise assessment of phase. Some more densely filled individual actograms (Figure 2) demonstrate that a daily onset and end of activity cannot be determined unambiguously. They further show that activity patterns do not differ conspicuously between genotypes. In fact, the same seasonal changes in Diurnality index (Figure 3) occur in all 3 genotypes simultaneously. The seasonal changes in environmental conditions leading to these shifts have far greater impact on the activity pattern than the presence or absence of a functional Per2 gene.

\section{Effect of Per2 Dysfunctionality on Fitness: Survival and Reproduction}

The data allowed us to assess survival of the genotypes in the population directly and of reproductive rates indirectly from the genotypic composition of the new cohorts with respect to what remained of the previous cohorts. We observed dramatic differences in the survival rates of the different genotypes, albeit in opposite direction in the 2 years of study. In the first year, homozygous mutants (Per2 ${ }^{\mathrm{mm}}$ ) suffered greater mortality than wild-types $\left(\right.$ Per $\left.2^{++}\right)$, with the heterozygotes $\left(\mathrm{Per}^{+\mathrm{m}}\right)$ in between, in both males and females. In the second year this pattern was reversed, although not significantly so in the males (Figure 4 and SOM-4). We have not been able to assess the causes of this differential mortality. Several deficits associated with the $\operatorname{Per} 2^{\text {Brdm } 1}$ mutation have been reported in the literature (reviewed by Albrecht et al., 2007). Homozygous Per ${ }^{\text {Brdm } 1}$ mutants have shown a failure to develop anticipatory activity to temporally restricted feeding times (Feillet et al., 2006) and to have increased resistance to lipopolysaccharide-induced endotoxic shock (Liu et al., 2006). Homozygous male mutant mice further appear to be more prone to tumor development and DNA damage following $\gamma$ radiation (Fu et al., 2002), consumption of addictive substances (Abarca et al., 2002; Spanagel et al., 2005), alterations in vascular endothelial function (Viswambharan et al., 2007), dipping of blood pressure and heart rate (Vukolic et al., 2010), and reduced endurance and strength of muscles (Albrecht et al., 2007; Bae et al., 2006). These or other effects may or not have played a role in the survival rate differentials we observed, but it is unlikely that circadian behavioral organization has been a key factor.

The comparison between genetic composition of the new cohorts with what remained from the old cohorts (Table 1) revealed that there was virtually no difference with respect to the Per 2 gene. We have therefore no basis for suggesting any deficit in reproduction as the basis for the change in gene frequency observed. Reproductive performance is known to be reduced in Per2-homozygous mutant middle-aged females (9-12 months), not in young adults (2-6 months) (Pilorz and Steinlechner, 2008). The proportion of females older than 6 months in the population can be coarsely estimated assuming a uniform distribution of birthdates of each cohort over the prior intercapture interval. This yields a figure of on average 
$31 \%$ of the female population older than 6 months. Since only $15 \%$ to $25 \%$ of these older females were homozygous mutants, it is understandable that the effect of such a reproductive skew must have been very minor indeed in the field and cannot be held responsible for the changes in gene frequency.

\section{Effect of Per2 Dysfunctionality on Fitness: Change in Gene Frequency}

We have seen (Figure 5A) how the mutant gene changed in frequency in the 4 populations studied. During the first summer nothing happened, but over the winter there was a general reduction in this frequency due to sharp decreases in 2 of the 4 pens and a slight reduction in a third pen. This change was first observed in the males starting in October 2005 and then a month later also in females. The overall frequency thereby dropped from 0.54 at the initial release in May 2005 to 0.41 in May 2006. From then on, the trend was reversed. While in the first year mice the mutant allele gradually disappeared further due to differential survival, the 2 new cohorts of 2006 (captured in May and August, respectively) increased steadily in Per $2^{\text {Brdm } 1}$ frequency (Figure 5C). Again, differential survival rather than differential reproduction caused this reversed direction of selection. The trend was observed in both pens ( 1 and 4 ) where the frequency had dropped in the first year, not in the third pen (3) where it had dithered around 50\% and then dropped in the second year. It is surprising that the many vital deficits known for Per2 mutants (Albrecht et al., 2007) apparently did not affect their fitness in conditions outdoors. The results indicate that these deficits would need more than 5 generations to make the allele disappear.

While the negative selection against the mutant allele in the first year was observed in both sexes, the increase in mutant allele frequency in the second year was primarily due to females (Figure 5B). By this time the sex ratio in the population had dropped to a stable level of around 25\% males (Figure 6). The sex ratio in homozygous mutants was much lower throughout, indeed zero during the late winter and spring of 2006. The strong positive effect of mutant alleles on median life span (>241 days in homozygote mutants compared to 64 days in wild-types) in the second year was observed solely in females (SOM-4).

The reversal of the change in allele frequency, and its dependence on the extent it had changed in the first year (Figure 5A), invites the following speculation. It is feasible that the individuals carrying 2 dysfunctional
$\operatorname{Per} 2^{\text {Brdm1 }}$ alleles that nonetheless stayed alive over the first winter were those that benefited from an otherwise stronger genomic background, which is a variable mixture of $\mathrm{C} 57 \mathrm{Bl}$ and 129 . Their offspring may have inherited this background and thereby gained an advantage in the second year. For example, there might exist, near the deleted locus of 129 origin, a second 129 specific allele amplifying negative effects of a deletion (Valenti et al., 2001). Traditional knockout techniques usually include a variable number of genes flanking the deleted locus (Wolfer et al., 2002). Continued segregation of 129 and C57 genes will eventually reduce the size of the flanking region, albeit unpredictably. Thus, mutants carrying only short chromosomal segments of 129 origin, more distant chromosomal positions being replaced with positive modifier genes from C57BL/6, would have a selective advantage.

It remains unclear why this or other advantages would only accrue to female mice. We must bear in mind, however, that the physiological deficits of Per $2^{\text {Brdm } 1}$ listed above and by Albrecht and colleauges (2007) were nearly all described in male mice and do not necessarily apply to females. Whatever the processes yielding the reversal in selection, we are in no position to invoke a role of the circadian system in the frequency of the Per $2^{\text {Brdm } 1}$ allele. On the contrary, impressive changes in circadian organization occur under seminatural conditions, which far exceed the effects of the presence or absence of functional Per2.

The study shows that gene frequency changes can be assessed in outdoor populations using automatic individual recording. Such systems will be applicable in studies of fitness impact of genomic variation. They may yield utterly surprising results, such as the diurnal activity of mice outdoors and the lack of impact on fitness in the field of a mutation that is deleterious in the lab.

\section{ACKNOWLEDGMENTS}

The study was supported by the EC-FP6 Integrated Project 018741 EUCLOCK (SD, UA), by grants from the Swiss National Science Foundation 3100A0-104222 and 3100A0-115984 (UA) and 31-108466 and 31-122589 (HPL); SCOPES IB74B0-111081, the Julius Klaus Stiftung für Genetik, and the NCCR program Neural Plasticity and Repair (HPL). We are indebted to Gerard Overkamp for help with raising and shipping the initial cohort of mice and to Vladimir and Natasha Bologov for dedicated assistance in the field. 


\section{NOTE}

Supplementary online material for this article is available on the Journal of Biological Rhythms website: http://jbr.sagepub.com/supplemental.

\section{REFERENCES}

Abarca C, Albrecht U, and Spanagel R (2002) Cocaine sensitization and reward are under the influence of circadian genes and rhythm. Proc Natl Acad Sci U S A 99: 9026-9030.

Abbott A (2007) A question of survival. Nature 449:532-534.

Albrecht U, Bordon A, Schmutz I, and Ripperger JA (2007) The multiple facets of Per2. Cold Spring Harb Symp Quant Biol 72:95-104.

Albrecht U, Zheng B, Larkin D, Sun ZS, and Lee CC (2001) mPer1 and mPer2 are essential for normal resetting of the circadian clock. J Biol Rhythms 16:100-104.

Bae K, Lee K, Seo Y, Lee H, Kim D, and Choi I (2006) Differential effects of two Period genes on the physiology and proteomic profiles of mouse anterior tibialis muscles. Mol Cells 22:275-284.

Calisi RM and Bentley GE (2009) Lab and field experiments: are they the same animal? Hormones Behav 56:1-10.

Comas M (2009) Entrainment to daylength in the mouse circadian system: behavioural and molecular analyses [dissertation]. University of Groningen.

Daan S and Tinbergen JM (1980) Young guillemots (Uria lomvia) leaving their arctic breeding cliffs: a daily rhythm in numbers and risk. Ardea 67:96-100.

DeCoursey PJ, Walker JK, and Smith SA (2000) A circadian pacemaker in free-living chipmunks: essential for survival? J Comp Physiol A Neuroethol Sens Neural Behav Physiol 186:169-180.

Feillet CA, Ripperger JA, Magnone MC, Dulloo A, Albrecht U, and Challet E (2006) Lack of food anticipation in Per2 mutant mice. Curr Biol 16:2016-2022.

Fu L, Pelicano H, Liu J, Huang P, and Lee CC (2002) The circadian gene Period 2 plays an important role in tumor suppression and DNA damage response in vivo. Cell 111:41-50

Gattermann R, Johnston R, Yigit N, Fritzsche P, Larimer S, Oezkurt S, Neumann K, Song Z, Colak E, Johnston J, et al. (2008) Golden hamsters are nocturnal in captivity but diurnal in nature. Biol Lett 4:253-255.

Hoogenboom I, Daan S, Dallinga J, and Schoenmakers M (1984) Seasonal change in the daily timing of behaviour of the common vole, Microtus arvalis. Oecologia (Berlin) 61:18-31.

Hut RA, Pilorz V, Boerema AS, Strijkstra AM, and Daan S (Forthcoming 2011) Working for food shifts nocturnal mouse activity into the day. PLOS One.

Liu J, Mankani G, Shi X, Meyer M, Cunningham-Runddles S, Ma X, and Sun ZS (2006) The circadian clock Period 2 gene regulates gamma interferon production of NK cells in host response to lippolysaccharide-induced endotoxic shock. Infect Immun 74:4750-4756.
Mendoza J, Albrecht U, and Challet E (2010) Behavioural food anticipation in clock genes deficient mice: confirming old phenotypes, describing new phenotypes. Genes Brain Behav 9:467-477.

Ouyang Y, Andersson CR, Kondo T, Golden SS, and Johnson CH (1998) Resonating circadian clocks enhance fitness in cyanobacteria. Proc Natl Acad Sci U S A 95: 8660-8664.

Perrigo G (1987) Breeding and feeding strategies in deer mice and house mice when females are challenged to work for their food. Anim Behav 35:1298-1316.

Pilorz V and Steinlechner S (2008) Low reproductive success in Per1 and Per2 mutant mouse females due to accelerated ageing? Reproduction 135:559-568.

Pittendrigh C (1960) Circadian rhythms and the circadian organization of living systems. Cold Spring Harb Symp Quant Biol, pp 159-184.

Pittendrigh CS and Daan S (1976) A functional analysis of circadian pacemakers in nocturnal rodents IV, Entrainment: pacemaker as clock. J Comp Physiol 106:291-331.

Pratt BL and Goldman BD (1986) Activity rhythms and photoperiodism of Syrian hamsters in a simulated burrow system. Physiol Behav 36:83-89.

Rowsemitt CN (1991) Activity rhythms in female montane voles (Microtus montanus). Can J Zool 69:1071-1075.

Schubert K, de Vries G, Vaanhol L, Meijer H, Daan S, and Verhulst S (2009) Maternal energy allocation to offspring increases with environmental quality in house mice. Am Nat 173:831-840.

Schubert KA, Boerema AS, Vaanholt LM, De Boer SF, Strijkstra AM, and Daan S (2010) Daily torpor in mice: high foraging costs trigger energy-saving hypothermia. Biol Lett 6:132-136.

Spanagel R, Pendyala G, Abarca C, Zghoul T, SanchisSegura C, Magnone MC, Lascorz J, Depner M, Holzberg D, Soyka M, et al. (2005) The clock gene Per2 influences the glutamatergic system and modulates alcohol consumption. Nat Med 11:35-42.

Spoelstra K, Albrecht U, van der Horst GTJ, Brauer V, and Daan S (2004) Phase responses to light pulses in mice lacking functional per or cry genes. J Biol Rhythms 19:518-529.

Spoelstra K and Daan S (2008) Effects of constant light on circadian rhythmicity in mice lacking functional cry genes: dissimilar from per mutants. J Comp Physiol A 194:235-242

Steinlechner S, Jacomeier B, Scherbarth F, Dernbach H, Kruse F, and Albrecht U (2002) Robust circadian rhythmicity of Per1 and Per2 mutant mice in constant light, and dynamics of Per1 and Per2 gene expression under long and short photoperiods. J Biol Rhythms 17:202-209.

Valenti P, Cozzio A, Nishida N, Wolfer DP, Sakaguchi S, and Lipp H-P (2001) Similar target, different effects: late-onset ataxia and spatial learning in Prion protein deficient mouse lines. Neurogenetics 4:173-184.

Van Zegeren K (1980) Variation in aggressiveness and the regulation of numbers in house mouse populations. Netherlands Journal of Zoology 30:635-770.

Viswambharan H, Carvas JM, Antic V, Marecic A, Jud C, Zaugg CE, Ming X-F, Montani J-P, Albrecht U, and Yang Z (2007) Mutation of the circadian clock gene Per2 alters vascular endothelial function. Circulation 115:2188-2195. 
Vukolic A, Antic V, Van Vliet BN, Yang Z, Albrecht U, and Montani J-P (2011) Role of mutation of the circadian clock gene Per2 in cardiovascular circadian rhythms. Am J Physiol Regul Integr Comp Physiol 298:R627-R634.

Vyssotski AL, Dell'Omo G, Poletaeva II, Vyssotski DL, Minichiello L, Klein R, Wolfer DP, and Lipp H-P (2002) Long-term monitoring of hippocampus-dependent behavior in naturalistic settings: mutant mice lacking neurotrophin receptor TrkB in the forebrain show spatial learning but impaired behavioral flexibility. Hippocampus 12:27-38.
Wolfer DP, Crusio WE, and Lipp H-P (2002) Knockout mice: simple solutions to the problems of genetic background and flanking genes. Trends Neurosci 25:336-340.

Zheng B, Albrecht U, Kaasik K, Sage M, Lu W, Vaishnav S, Li Q, Sun ZS, Eichele G, Bradley A, and Lee CC (2001) Nonredundnt roles of the mPer1 and mPer2 genes in the mammalian circadian clock. Cell 105:683-694.

Zheng B, Larkin DW, Albrecht U, Sun ZS, Sage M, Eichele G, Lee CC, and Bradley A (1999) The mPer2 gene encodes a functional component of the mammalian circadian clock. Nature 400:169-173. 